\title{
Implicit large-eddy simulations of zero-pressure gradient, turbulent boundary layer
}

\author{
Susheel Sekhar* and Nagi N. Mansour ${ }^{\dagger}$ \\ NASA Ames Research Center, Moffett Field, CA 94035
}

\begin{abstract}
A set of direct simulations of zero-pressure gradient, turbulent boundary layer flows are conducted using various span widths (62-630 wall units), to document their influence on the generated turbulence. The FDL3DI code that solves compressible Navier-Stokes equations using high-order compact-difference scheme and filter, with the standard recycling/rescaling method of turbulence generation, is used. Results are analyzed at two different $\boldsymbol{R e}_{\theta}$ values (500 and 1,400), and compared with spectral DNS data. They show that a minimum span width is required for the mere initiation of numerical turbulence. Narrower domains ( $<100 \mathrm{w.u}$.) result in relaminarization. Wider spans ( $>600$ w.u.) are required for the turbulent statistics to match reference DNS. The upper-wall boundary condition for this setup spawns marginal deviations in the mean velocity and Reynolds stress profiles, particularly in the buffer region.
\end{abstract}

\section{Introduction}

Turbulent boundary layers have been the subject of intensive research for many decades, partly because of their pervasive nature. With advances in computing resources, extending the state-of-the-art in understanding these complex flows has been further enabled.

Ever since Spalart's seminal paper, ${ }^{1}$ in which he presented DNS of turbulent boundary layer on a flat plate for a range of $\operatorname{Re}_{\theta}$ values $(225-1,410)$, successive efforts have managed to extend this high Reynolds number limit. Rai and Moin $^{2}$ used a high-order finite-difference approach to study transition and turbulence of a spatially evolving boundary layer up to $\operatorname{Re}_{\theta}=2,300$. Ferrante and Elghobashi ${ }^{3}$ used the recycling/rescaling technique to study spatially developing incompressible boundary layers up to $\operatorname{Re}_{\theta}=1,430$.

More recently, a number of research efforts have made significant advances in extending that limit further. Lee and Sung ${ }^{4}$ also used a similar technique as this paper, and computed turbulent boundary layer up to $\operatorname{Re}_{\theta}=2,500$. Schlatter and Örlü were able to further extend DNS data to $\operatorname{Re}_{\theta}=4,300$ using spectral methods. Within the last few years, Sillero et al. ${ }^{6}$ managed to breach the 6,000 limit and generate results up to $\operatorname{Re}_{\theta}=6,650$. Borrell et al. ${ }^{7}$ furthered this by computing up to $\operatorname{Re}_{\theta}=6,800$.

All of these efforts have been restricted to the incompressible regime, and many of them used spectral methods. The applicability of such techniques to practical flow situations is rather limited. Not necessarily unique, this study steps outside that usual realm by using a finite-difference code that solves compressible Navier-Stokes equations. Documenting challenges encountered, and subsequent solutions, will be useful for future simulations of compressible turbulent flows. This study reports the requirements for accurate predictions of turbulent boundary layers, including the minimum spanwise width of the domain. In that sense, this work follows Jiménez and Moin's article on the impact of width on turbulent channel flow. ${ }^{8}$ In their study, they conducted DNS of low Reynolds number channel flow to study the morphology and dynamics of the most basic flow unit of turbulence. The fundamental period of intermittency was identified, and the impact of different domain widths on the growth of turbulence was investigated for the unsteady channel flow problem. This study analyzes turbulent boundary layer on a flat plate instead.

This study is a part of an overall effort to understand turbulent, separated flows. There continues to be a dearth in the physics knowledge-base for such phenomena. This is reflected in the computations of separated flows using RANS models, which significantly deviate from experimental observations, particularly

* NASA Postdoctoral Program Fellow, AIAA Member

${ }^{\dagger}$ Chief Division Scientist, NASA Advanced Supercomputing Division, AIAA Associate Fellow 
in reattachment and recovery. The understanding that this study presents will aid to further improve the state-of-the-art in flow predictions using relatively inexpensive RANS and hybrid RANS/LES methods. As a precursory exercise, it is important to establish the capacity to predict attached turbulent boundary layers at various Reynolds numbers. In the process, requirements for accurate predictions of the physics are parameterized. Included amongst these are the minimum width of span and the ideal mesh resolution at the wall. Results from this effort are being used in a simultaneous study of turbulent, separated flows past a backward-facing step and a wall-mounted hump. They are also being used to set up simulations at higher $\operatorname{Re}_{\theta}$ values.

The paper is structured as follows: Section II briefly explains the numerical methodology used for the simulations. Section III documents the results for both $\operatorname{Re}_{\theta}=500$ and 1,400 simulations. Conclusions are drawn in Sec. IV.

\section{Numerical Method}

\section{II.A. Governing Equations, Compact Difference Scheme and Filter}

The finite difference code (a variant of AFRL's FDL3DI ${ }^{9,10}$ ) used in this study solves the compressible NavierStokes equations for an ideal gas. The sixth-order compact-difference scheme of Lele ${ }^{11}$ is used to solve the governing equations in transformed curvilinear coordinates. ${ }^{12,13}$ Fourth-order explicit Runge-Kutta (RK-4) is used for time integration. During each time step, an eighth-order low-pass spatial filtering scheme is applied to the conservative variables to ensure stability, along with second- and fourth-order near-boundary formulations. ${ }^{14}$ A filtering optimization parameter of $\alpha_{f}=0.495$ is set. To ensure stability and accuracy of time integration, a Courant-Friedrichs-Lewy (CFL) number in the vicinity of 0.4 , based on the acoustic speeds, is used in the RK-4 scheme.

This code was previously designed to solve for supersonic flows, specifically those involving shock-wave and boundary layer interaction problems. Therefore, the localized artificial diffusivity (LAD) method of shock-capturing was previously installed by Kawai et al., ${ }^{15,16}$ and was used in a detailed study of the flow physics of such interactions by Morgan et al. ${ }^{17}$ This method adds artificial coefficients to the fluid transport terms, and takes effect in the vicinity of shocks. Since the flow regime under consideration is subsonic, LAD is not included in the current study. Thus, other than the low-pass filter, no additional sub-grid scale model is used. In that sense, this effort can be considered implicit-LES, or more precisely "under-resolved DNS."

\section{II.B. Recycling/Rescaling Turbulence Inflow Method}

For generating the turbulent inflow, the standard recycling/rescaling method of Lund et al. ${ }^{18}$ is used in the code. Urbin and Knight ${ }^{19}$ extended this method to compressible flows for solving a Mach 3 turbulent boundary layer. This was implemented as a part of the effort to study the interaction of an oblique shock wave and a turbulent boundary layer by Morgan et al. ${ }^{20}$ The approach extracts the instantaneous velocity profile from a plane downstream of the inflow, rescales and reintroduces it as the inflow. The schematic of the procedure is shown in Fig. 1. It has been shown to produce a realistic turbulent boundary layer, yielding statistics that are in good agreement with experiment and DNS. To further prevent contamination of the solution with spurious spatio-temporal correlations generated by this reintroduction procedure, a nonconstant reflection of the recycled turbulence plane is applied at randomly distributed time intervals. ${ }^{20}$ This method, called recycling/rescaling with dynamic reflection $(\mathrm{RR}+\mathrm{DR})$, has been suitably adjusted to solve subsonic compressible flows.

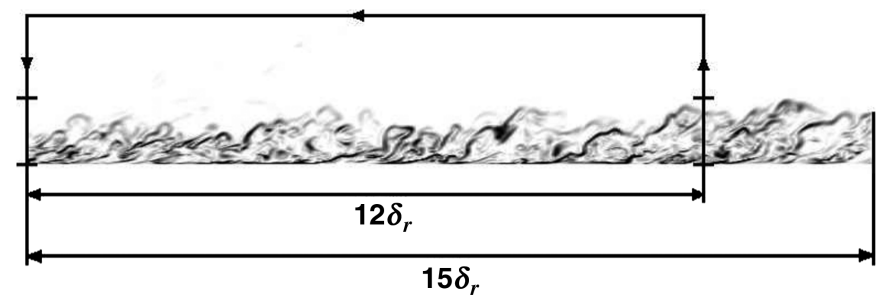

Figure 1. Recycling-rescaling methodology schematic ${ }^{21}$ 


\section{Results}

The setups for validating the code for subsonic turbulent boundary layers are detailed here. A separate simulation was required for each $\operatorname{Re}_{\theta}$.

For both simulations, a domain of streamwise length $15 \delta_{\mathrm{R}}$ and height $3 \delta_{\mathrm{R}}$ was used, where $\delta_{\mathrm{R}}$ is the desired boundary layer thickness at the velocity capture plane. This capture plane was located about $12 \delta_{\mathrm{R}}$ downstream of the inlet (Fig. 1), and was also the location of the target $\operatorname{Re}_{\theta}$. The initial mesh in each case had a spanwise width of $\frac{1}{4} \delta_{\mathrm{R}}$, and this was doubled successively as required. This mesh consisted of $500 \times 100 \times 27$ points, and was uniformly spaced along the streamwise and spanwise directions. A hyperbolic tangent stretching was set in the wall normal direction.

For the boundary conditions, the inlet was computed during the recycling/rescaling procedure, and a freestream pressure outflow was set for the outlet. A no-slip, adiabatic wall was used for the bottom wall. First-order extrapolation of the state variables was performed at the top wall. Along the spanwise direction, periodic boundary conditions were used.

For both cases, sea level conditions were assumed, with a freestream Mach number of 0.3 . A $0.1 \%$ random perturbation was included as a part of the initial condition.

\section{III.A. $\boldsymbol{R e}_{\theta}=500$}

To simulate $\operatorname{Re}_{\theta}=500$ at the location $12 \delta_{\mathrm{R}}$ downstream of the inlet, $\operatorname{Re}_{\theta}$ at the inlet was set to 350 , based on Prandtl's one-seventh power law approximation .

At these flow conditions, the mesh resolution at the wall was computed to be $\Delta x^{+}=7.5$ and $\Delta z^{+}=2.3$, along the streamwise and spanwise directions, respectively. The overall spanwise width of the domain was $L_{z}=62$ w.u. In the wall normal direction, the spacing was $\Delta y^{+}=0.25$ at the wall, and was stretched using a hyperbolic-tangent function. With such a resolution, this mesh is almost twice as fine as that used by Spalart for his spectral DNS. But, as Moin and Mahesh noted, ${ }^{22}$ spatial resolution requirements are dictated by the choice of numerical schemes. They showed that sixth-order Padè requires $60 \%$ finer meshes than those demanded in Fourier spectral methods.

Flow development was monitored in terms of $u$ velocity component contours, to determine the initialization of turbulence. As shown in the time series in Fig. 2, after approximately 12 flow through time cycles (FTTs), the laminar nature of the boundary gradually starts to break down. After about 15 FTTs, wavy Kelvin-Helmholtz-like instabilities are evident in the domain. These disturbances, by means of the recycling/rescaling process, are fed back into the domain, resulting in an increase in their intensity. This process continues to perturb the flow until about 22 FTTs, when the flow starts to go turbulent. At this point, instead of sustaining the turbulent motion in the domain, the flow relaminarizes and the disturbances get dampened out. It should be noted that this phenomenon is a purely numerical transition because of the recycling process, and does not correspond to the physical transition from a laminar to a turbulent profile.

A similar observation was made by Jiménez and Moin $^{8}$ for channel flows, where domains with spanwise widths lesser than a threshold failed to sustain turbulent motion. In their estimate, for spans narrower than 100 w.u., laminar solutions alone were found. This observation is in agreement with their estimate. Periodicity on such narrow domains compromises 2-point correlations, damping out the initiated turbulence. To confirm this, the $L_{z}=62$ w.u. domain was doubled along the span, and the simulation was continued.

This new mesh consisted of $500 \times 100 \times 53$ points, and the initial condition corresponded to the steady solution from the previous simulation, replicated along the span. $u$ contours were once again monitored, and the time series for this setup is shown in Fig. 3

Similar to the previous setup, instabilities in the flow start to appear immediately, and after around 3 FTTs, the flow starts to undergo numerical transition. The recycling/rescaling feed back process continues to increase the intensity of perturbations, which unlike in the narrower domain, become self-sustaining. Even after 15 FTTs, the perturbations are sustained, indicating that the flow has taken up a turbulent nature.

To evaluate the response to even wider spans, the domain was once again doubled, resulting in a mesh with $500 \times 100 \times 105$ points. The resulting $L_{z}=240$ w.u. The domain was initialized in a similar manner, using the statistically steady data from the $L_{z}=122$ w.u. simulation. The resulting mean velocity profiles at $x=12 \delta_{\mathrm{R}}$ location are shown in Fig. 4 . The relaminarized profile is indeed thinner than the two turbulent profiles, which have higher momentum content at lower $y / \delta$ locations.

Having established the turbulent nature qualitatively, statistical averages of the velocity profile (Fig. 5) and Reynolds stresses (Fig. 6) were compared with DNS results of Spalart. ${ }^{1}$ These were computed at the 


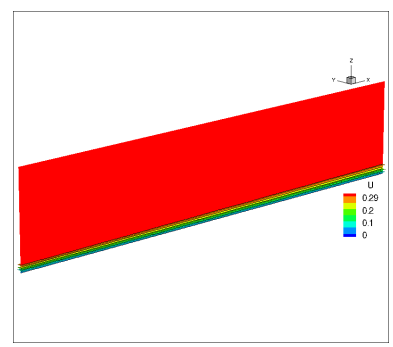

(a) 12 FTTs

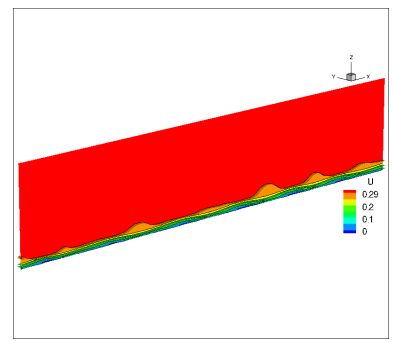

(e) 18 FTTs

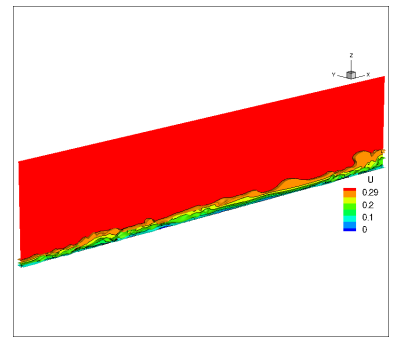

(i) $24 \mathrm{FTTs}$

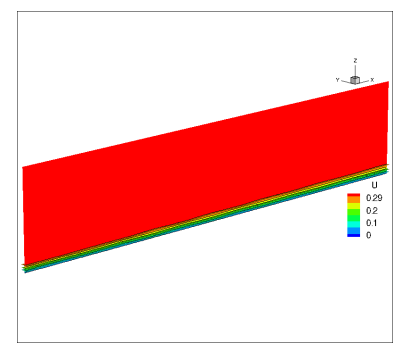

(b) 13 FTTs

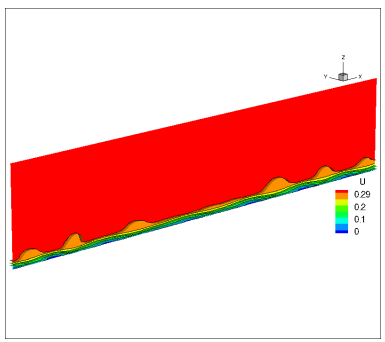

(f) 19 FTTs

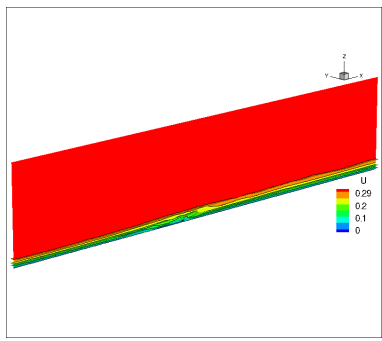

(j) $25 \mathrm{FTTs}$

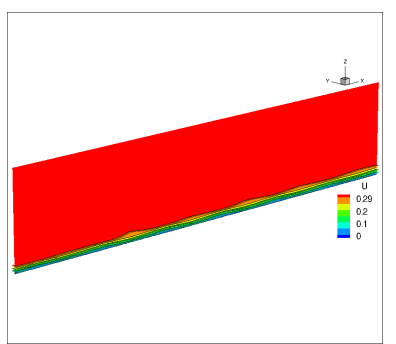

(c) 15 FTTs

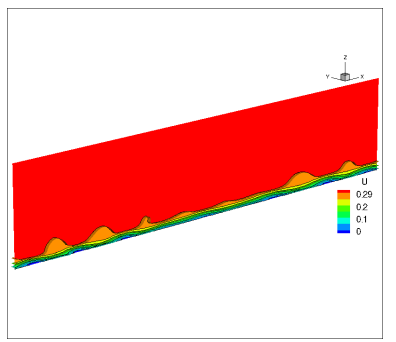

(g) 21 FTTs

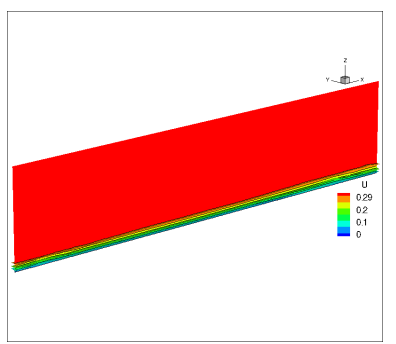

(k) 26 FTTs

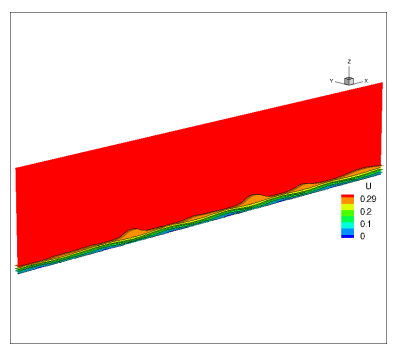

(d) 16 FTTs

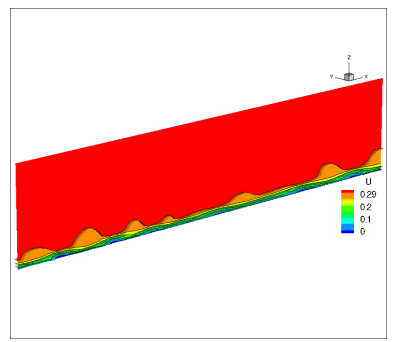

(h) 22 FTTs

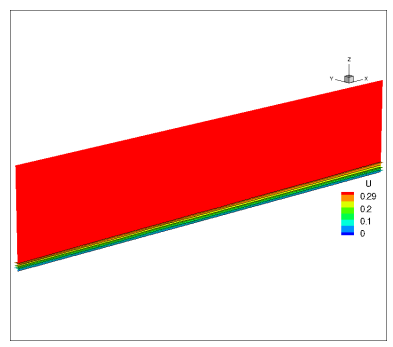

(1) 27 FTTs

Figure 2. Time series of $\mathbf{u}\left(x_{i}, t\right)$ contours for a period of 15 flow-through times (FTTs) showing the apparent transition from laminar to a numerically turbulent flow, and subsequent relaminarization, for $L_{z}=62 \mathrm{w} \cdot \mathrm{u}$.

$12 \delta_{\mathrm{R}}$ location, where the simulated $\operatorname{Re}_{\theta}=500$. Data was averaged along the spanwise direction, as well as over a number of flow through time cycles.

To get an estimate on where the current simulation falls in comparison with the reference, spectral DNS mean velocity profiles at three different $\operatorname{Re}_{\theta}$ locations $(300,670,1,410)$ are plotted in Fig. 5 (a), along with the results from the implicit LES simulations. All three widths show perfect agreement within the linear viscous sublayer, until $y^{+} \approx 7$. For the domain with $L_{z}=62$ w.u., the laminar profile is distinctly devoid of buffer and log layers thereafter, in addition to being offset in terms of the magnitude of the freestream. The turbulent profiles with $L_{z}=122$ and $240 \mathrm{w} . \mathrm{u}$. wide domains were averaged over 20 FTTs, and more reasonable agreements are evident. The buffer and log layers become apparent, though the freestream magnitude is still relatively large. Whereas the ideal freestream for $\operatorname{Re}_{\theta}=500$ profile should lie between the $R e_{\theta}=300$ and 670 curves, that for $L_{z}=122 \mathrm{w} . \mathrm{u}$. falls between $R e_{\theta}=670$ and 1 , 410 curves, and the $L_{z}=240 \mathrm{w} . \mathrm{u}$. freestream matches $R e_{\theta}=670$. These differences are attributed to the boundary condition set at the upper wall of the domain. By extrapolating the state variables, the condition at the upper wall is similar to that of slip-wall or symmetry boundary, and comparisons of the mean profiles do demonstrate that (not shown). Such a boundary condition causes the freestream to accelerate, resulting in higher mean velocities.

Slight differences between spectral DNS and implicit LES are also evident within the buffer layer. This region is magnified in Fig. 5 (b). $u^{+}$for both $L_{z}=122$ and $240 \mathrm{w} . u$. are marginally higher than the reference flat plate profiles of Spalart. This, again, is attributed to the symmetry-like boundary condition at the upper wall, which essentially makes the current results similar to that of a channel flow. For comparison, channel results of Moser et al. ${ }^{23}$ are also shown in the figure, and there is better agreement of the computed buffer layer with them. 


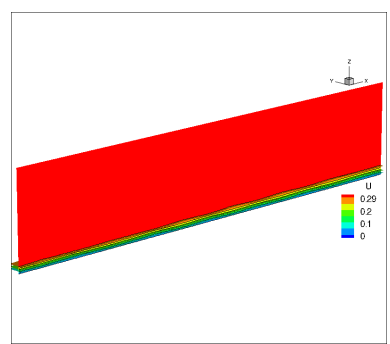

(a) Initial

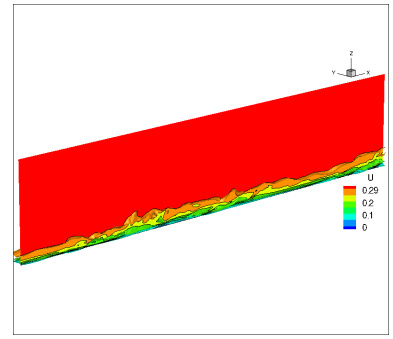

(e) 6 FTTs

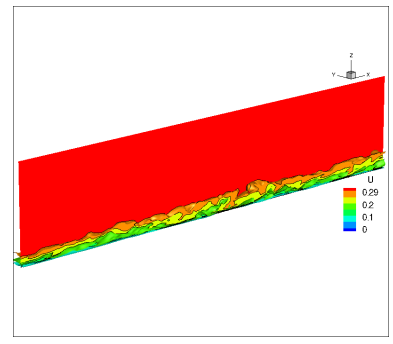

(i) 12 FTTs

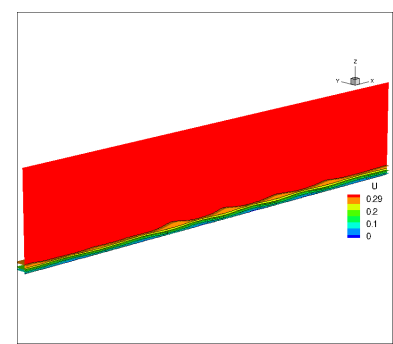

(b) 1 FTTs

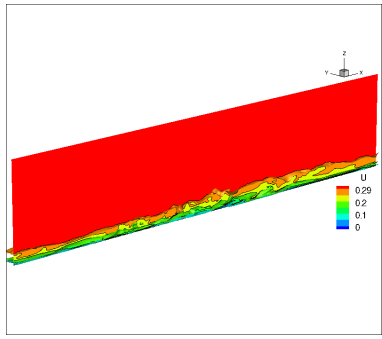

(f) 7 FTTs

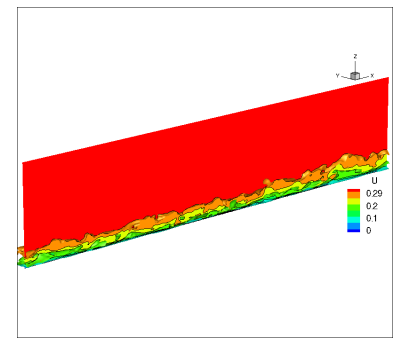

(j) 13 FTTs

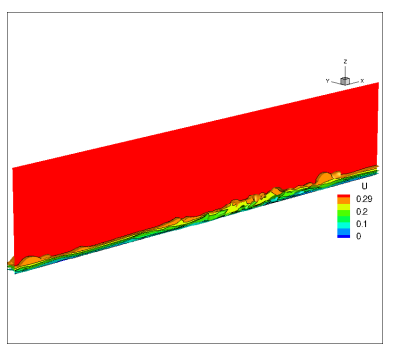

(c) 3 FTTs

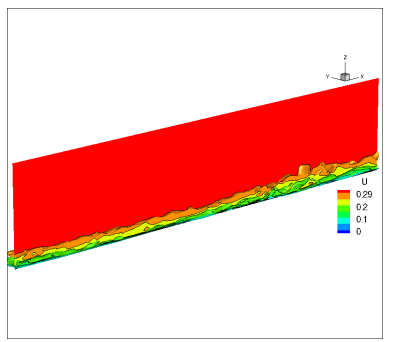

(g) 9 FTTs

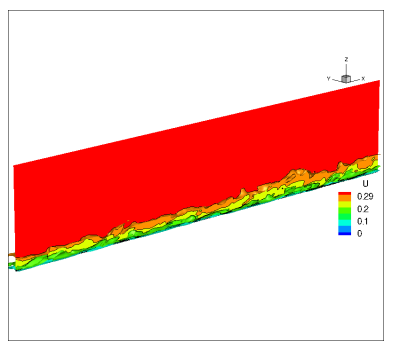

(k) 14 FTTs

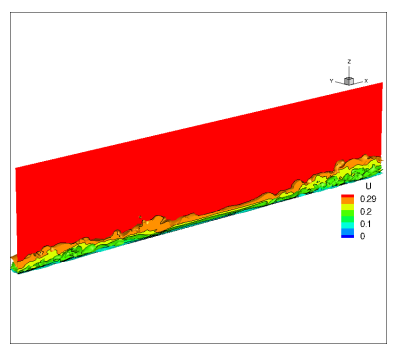

(d) 4 FTTs

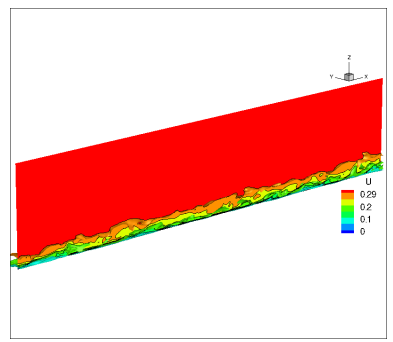

(h) 10 FTTs

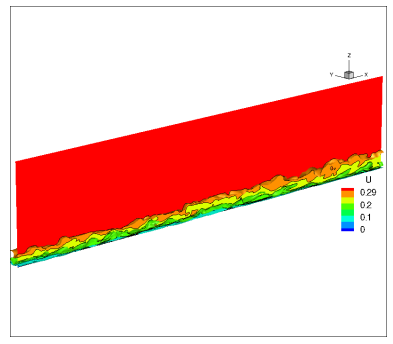

(1) 15 FTTs

Figure 3. Time series of $\mathbf{u}\left(x_{i}, t\right)$ contours for a period of 15 FTTs showing the apparent transition from laminar to a numerically turbulent flow, for $L_{z}=122 \mathrm{w} . u$.

Reynolds stresses comparisons are shown in Fig. 6. $\langle u u\rangle,\langle v v\rangle,\langle w w\rangle$ and $\langle u v\rangle$ averages for $L_{z}=122$ and 240 w.u. are compared with reference DNS data. Since the $L_{z}=62$ w.u. case relaminarized, that is not shown here. For $L_{z}=122$ w.u., deviations from the references values are more pronounced, both, within the buffer layer and away from the wall (log layer). $\langle u u\rangle$ and $\langle v v\rangle$ fare poorly throughout the boundary layer, and it is concluded that the generated turbulence is non-physical for this span width. There is improved agreement for $L_{z}=240$ w.u., but since spectral DNS for $R e_{\theta}=500$ is unavailable, precise conclusions could not be drawn. Nevertheless, statistics within the viscous sublayer and the log layer show significant improvements, but there are differences evident within the buffer layer, especially for $<u u>$ and $<v v>$.

III.B. $\quad \boldsymbol{R e}_{\theta}=1,400$

Since spectral DNS data for $\operatorname{Re}_{\theta}=1,410$ is available, a more definitive comparison could be made for this case. To simulate $\operatorname{Re}_{\theta}=1,400$ at a location $12 \delta_{\mathrm{R}}$ downstream of the inlet, $\operatorname{Re}_{\theta}$ at the inlet was set at 1,048 , based on Prandtl's one-seventh power law approximation.

Here again, an initial mesh of $500 \times 100 \times 27$ points was considered. Based on the flow conditions, mesh resolution at the wall was computed to be 19.4 w.u. and 6 w.u. in the streamwise and spanwise directions, respectively. In the wall normal direction, the spacing was 0.65 w.u. at the wall, and was again stretched using a hyperbolic-tangent function. The overall span width was $L_{z}=162 \mathrm{w} . \mathrm{u}$. This is comparable to the resolution using by Spalart for his spectral DNS.

At this width, transition to numerical turbulence occurred with the initial grid itself after approximately 18 FTTs. Similar to the lower $\operatorname{Re}_{\theta}$ case, this initial domain was doubled twice along the span, resulting in 


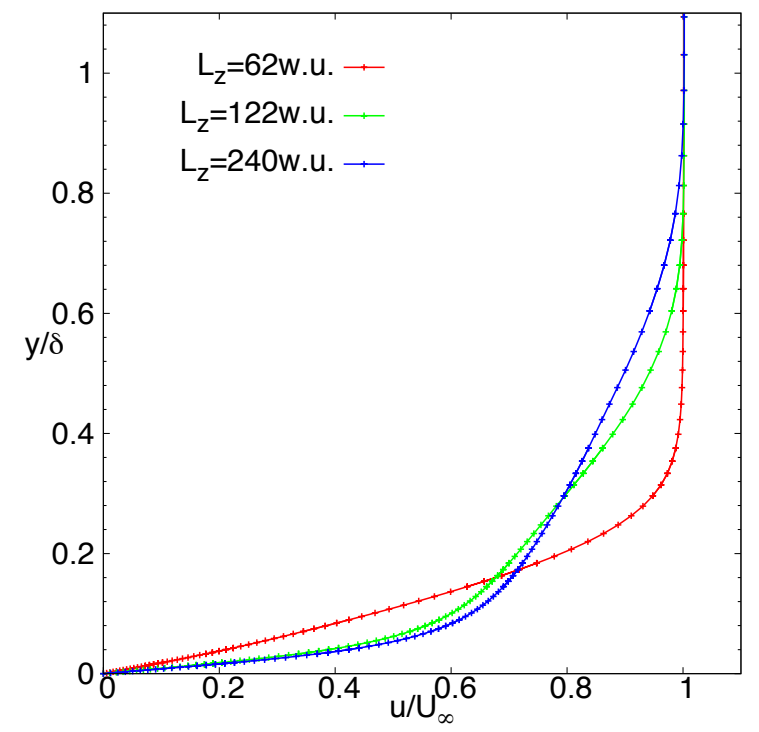

Figure 4. Mean velocity profile for different span widths, at $\mathbf{R e}_{\theta}=500$.
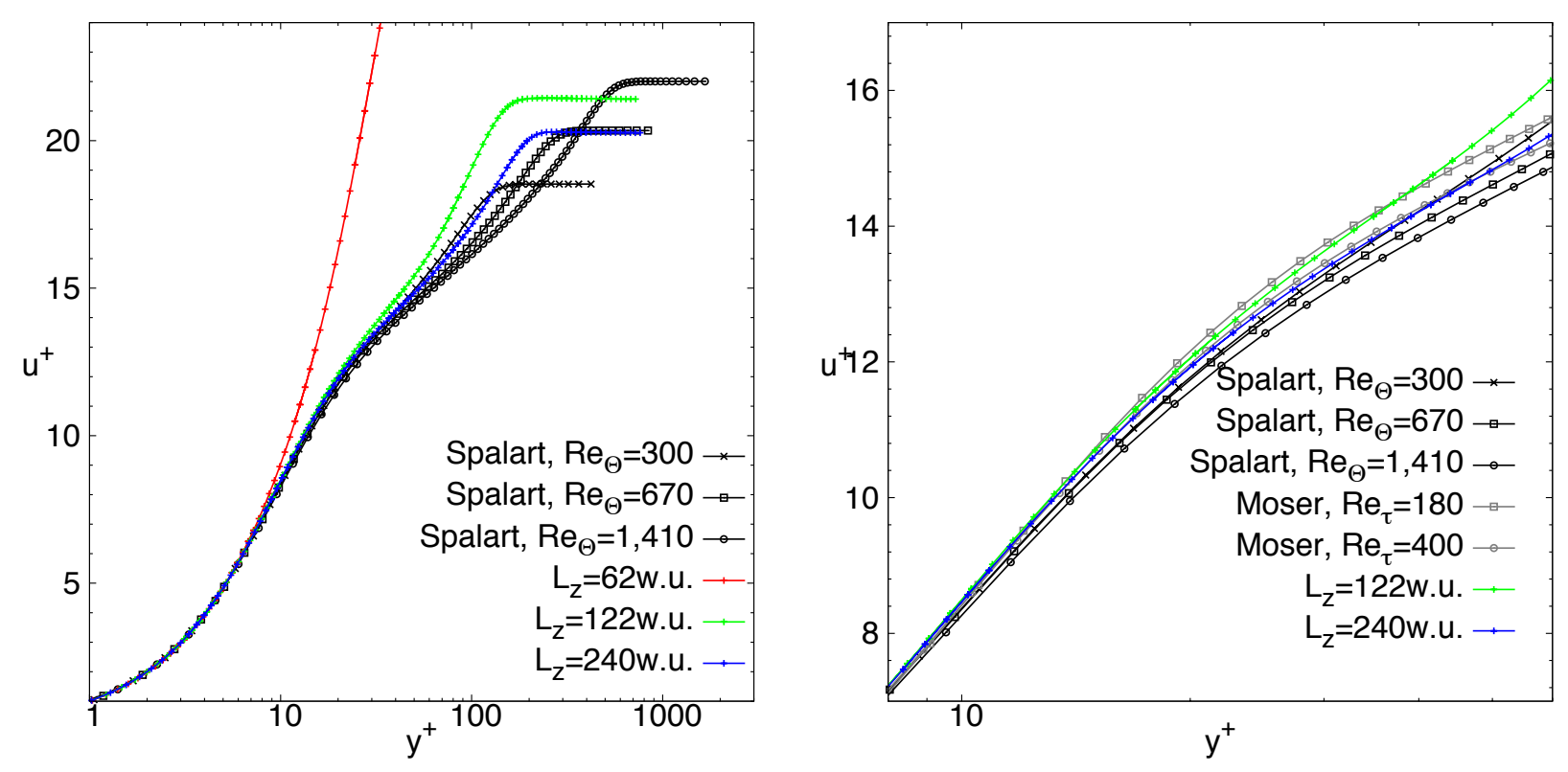

(a) Viscous sub-layer, buffer layer and log-law region

(b) Buffer layer, magnified

Figure 5. Mean velocity profile in wall units for different span widths, at $\mathbf{R e}_{\theta}=500$, compared with DNS results for flat plate of Spalart, ${ }^{1}$ and channel flow of Moser et al. ${ }^{23}$ 


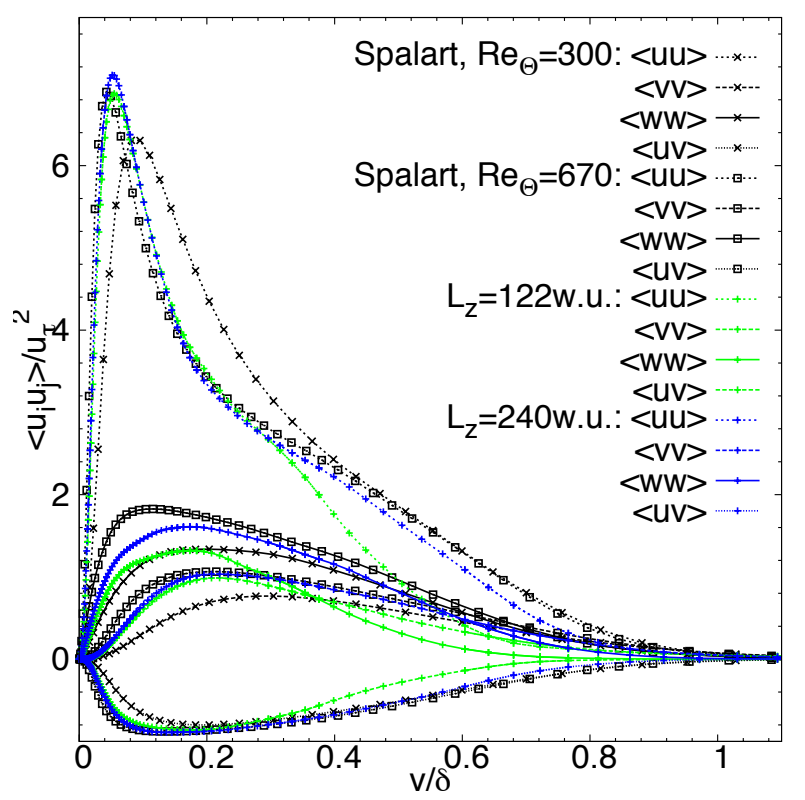

(a) Across the boundary layer

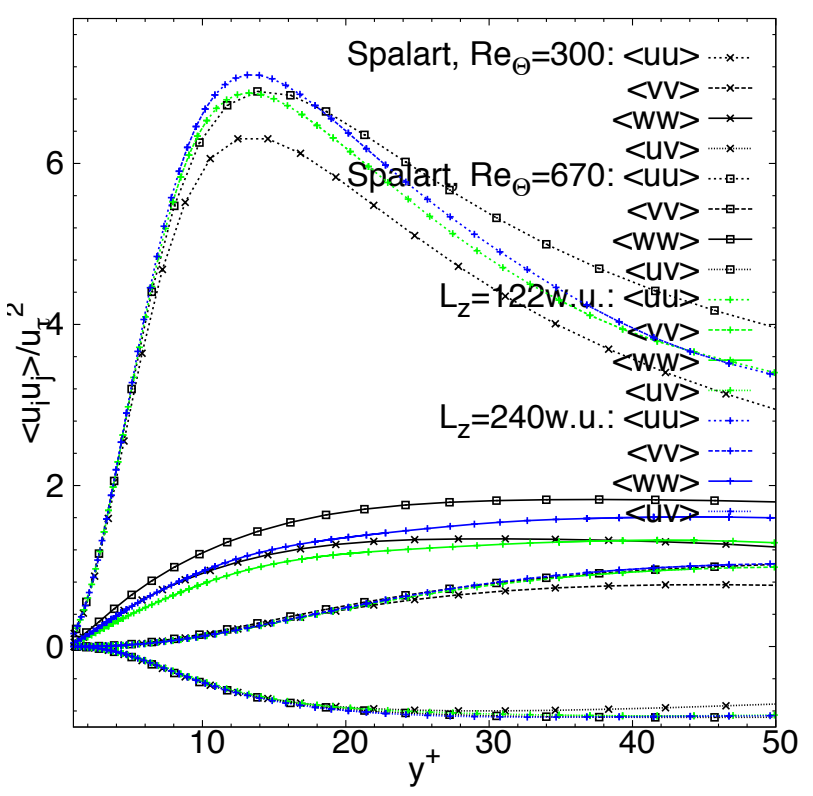

(b) In the viscous near-wall region

Figure 6. Reynolds stresses for different span widths, normalized by friction velocity, at Re $\theta=500$, compared with DNS results of Spalart ${ }^{1}$

domains that had 318 and 630 w.u. spans, respectively. The mean velocity profiles at the $12 \delta_{\mathrm{R}}$ location are shown in Fig. 7. That the boundary layer is thicker with increasing span widths is evident, with the mean velocities tending towards an asymptotic profile.

Statistical averages of the mean velocity (Fig. 8) and Reynolds stresses (Fig. 9) at the $12 \delta_{\mathrm{R}}$ location are compared with reference DNS. Data was averaged over 20 FTTs for each setup, and statistical stationarity of the solution was established.

In Fig. 8 (a), all three spans show evidence of the viscous sublayer, buffer layer and the log layer, unlike the previous case. There is, once again, very good agreement with spectral DNS for the viscous sublayer, until $y^{+} \approx 10$. The freestreams for all three cases are over-predicted, progressively dropping in magnitudes as the domains are widened. Deviations within the buffer layer are clearly evident here. This region is magnified in Fig. 8 (b), where spectral DNS of channel flow of Moser et al. ${ }^{23}$ are also included for clarity. The agreement of the current implicit LES with channel data can be clearly seen, confirming that the upper-wall boundary condition indeed influences the buffer zone. The acceleration of the freestream is also attributed to this symmetry-like boundary condition.

For an ideal boundary layer setup, the top wall must accommodate the displacement in the freestream due to the presence of the boundary layer. A simple extrapolation does not sufficiently account for that displacement thickness. A vertical velocity profile, similar to that used by Lund et al., ${ }^{18}$ determined from the integrated continuity relation, $v(x)=U_{e} d \delta^{*} / d x+\left(\delta^{*}-h\right) d U_{e} / d x$, should accommodate for that displacement thickness. In this study, since these are auxiliary simulations to channel-like configurations of separated flows, that boundary condition was not investigated.

Comparisons of Reynolds stresses with reference DNS are shown in Fig. 9. Overall agreement significantly improves with increasing spanwise widths, with $L_{z}=630 \mathrm{w}$.u. showing excellent agreement for $\langle v v\rangle,\langle w w\rangle$ and $\langle u v\rangle$ profiles throughout the boundary layer. There is an over-prediction of the peak $\langle u u\rangle$ stress, which lies within the buffer zone, attributed once again to the upper-wall boundary condition. The influence of the higher peak $\langle u u\rangle$ is evident throughout the entire boundary layer. 


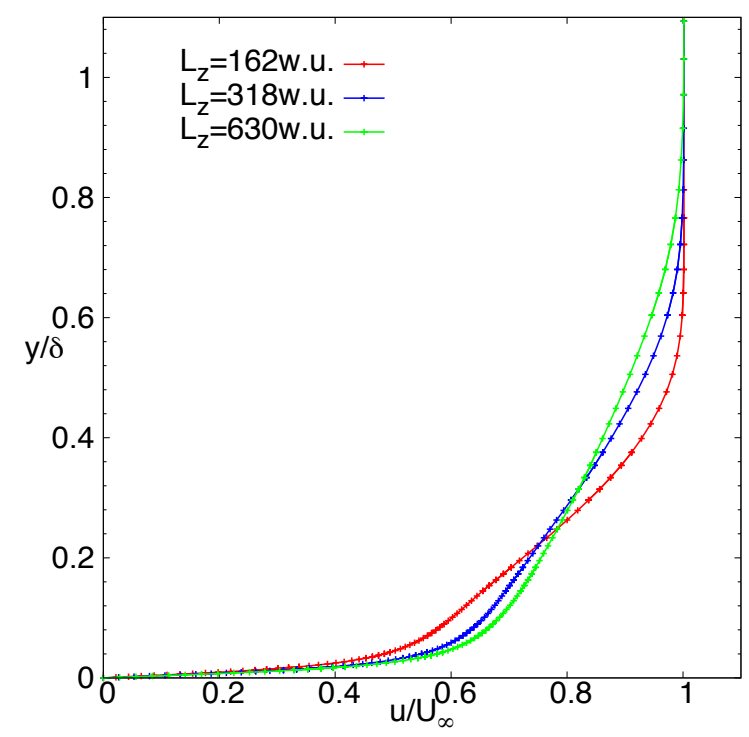

Figure 7. Mean velocity profile for different span widths, at $\mathbf{R e}_{\theta}=1400$.

\section{Conclusions}

Direct simulations of zero-pressure gradient, turbulent boundary layers for two separated $\operatorname{Re}_{\theta}$ values (500 and 1,400) were conducted, and compared with spectral DNS data. The high-order compact-difference scheme and filter, with the standard recycling/rescaling method of turbulence generation, used meshes with similar resolutions as DNS, and the impact of varying span widths was investigated.

A minimum span width was required for the mere initiation and continuation of Kelvin-Helmholtz-like numerical instabilities. Narrower domains $(<100$ w.u.) failed to sustain the perturbations, resulting in relaminarization. Wider spans did sustain the instabilities. Comparisons of mean velocity profiles and Reynolds stresses showed that a span of $630 \mathrm{w} . \mathrm{u}$. was required for the turbulent statistics to match reference DNS. The upper-wall boundary condition for this setup generated marginal deviations in the mean velocity and Reynolds stress profiles, particularly in the buffer zone. A symmetry-like upper-wall resulted in better agreement with channel data within the buffer zone, and a marginally faster freestream.

\section{Acknowledgements}

This research was supported by an appointment to the NASA Postdoctoral Program at Ames Research Center, administered by Oak Ridge Associated Universities through a contract with NASA. The authors are grateful for the support of NASA's Fundamental Aeronautics Program through the Aeronautical Sciences Project.

\section{References}

${ }^{1}$ Spalart, P. R., "Direct simulation of a turbulent boundary layer up to $\operatorname{Re}_{\theta}=1410$," Journal of Fluid Mechanics, Vol. 187 , 1988, pp. 61-98.

${ }^{2}$ Rai, M. M. and Moin, P., "Direct Numerical Simulation of Transition and Turbulence in a Spatially Evolving Boundary Layer," Journal of Computational Physics, Vol. 109, 1993, pp. 169-192.

${ }^{3}$ Ferrante, A. and Elghobashi, S. E., "A robust method for generating inflow conditions for direct simulations of spatiallydeveloping turbulent boundary layers," Journal of Computational Physics, Vol. 198, 2004, pp. 372-387.

${ }^{4}$ Lee, J. H. and Sung, H. J., "Direct numerical simulation of a turbulent boundary layer up to $\operatorname{Re}_{\theta}=2500$," International 


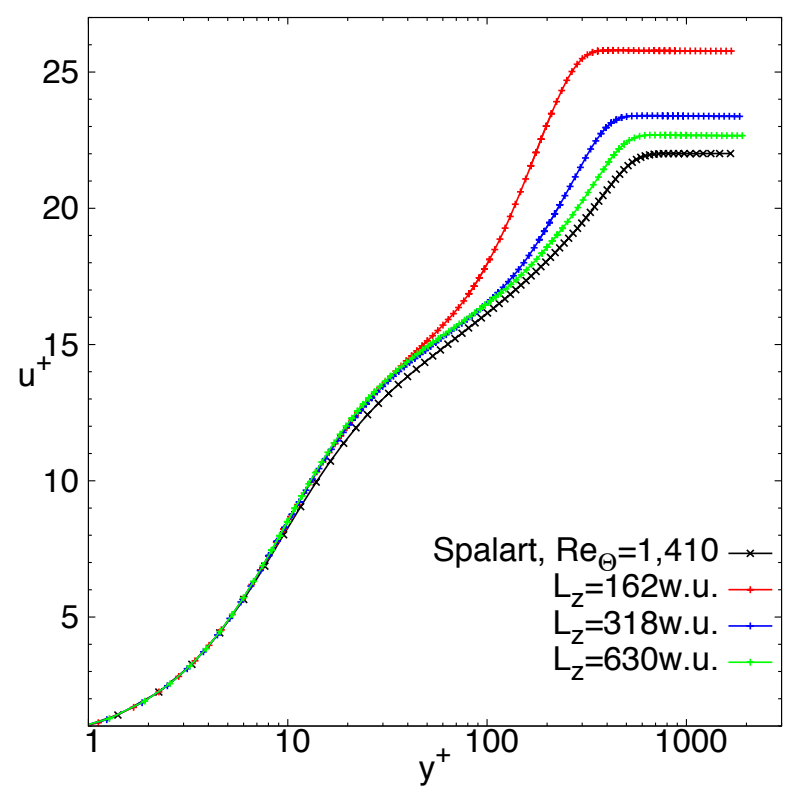

(a) Viscous sub-layer, buffer layer and log-law region

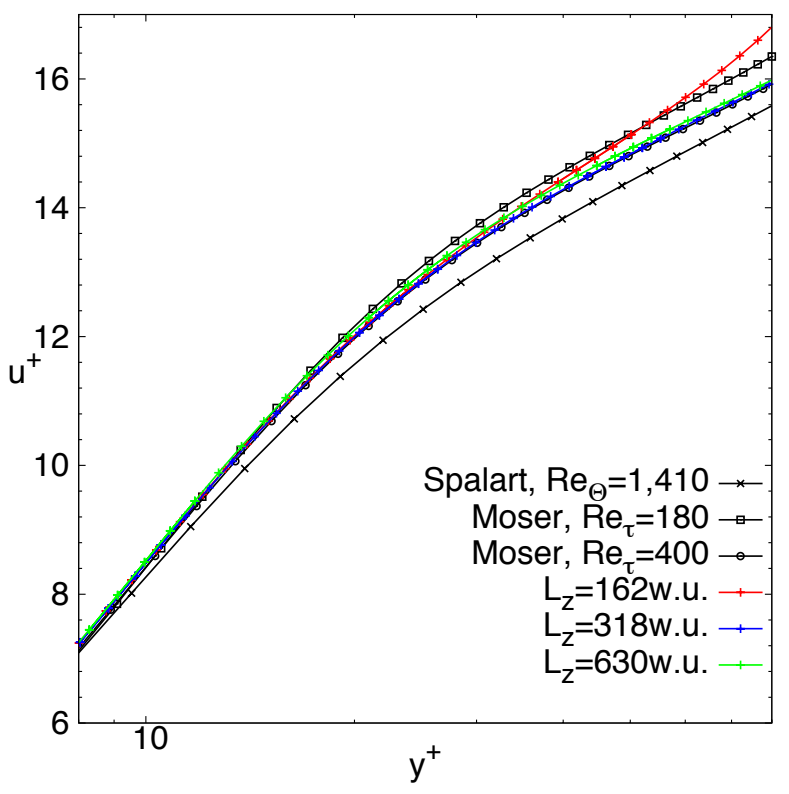

(b) Buffer layer, magnified

Figure 8. Mean velocity profile in wall units for different span widths, at $\mathbf{R e}_{\theta}=1400$, compared with DNS results for flat plate of Spalart, ${ }^{1}$ and channel flow of Moser et al. ${ }^{23}$

Journal of Heat and Fluid Flow, Vol. 32, 2011, pp. 1-10.

${ }^{5}$ Schlatter, P. and Örlü, R., "Assessment of direct numerical simulation data of turbulent boundary layers," Journal of Fluid Mechanics, Vol. 659, 2010, pp. 116-126.

${ }^{6}$ Sillero, J., Jiménez, J., Moser, R. D., and Malaya, N. P., "Direct simulation of a zero-pressure-gradient turbulent boundary layer up to $\operatorname{Re}_{\theta}=6650$," Journal of Physics: Conference Series, Vol. 318, No. 022023, 2011.

${ }^{7}$ Borrell, G., Sillero, J. A., and Jiménez, J., "A code for direct numerical simulation of turbulent boundary layers at high Reynolds numbers in BG/P supercomputers," Computer 8 Fluids, Vol. 80, 2013, pp. 37-43.

${ }^{8}$ Jiménez, J. and Moin, P., "The minimal flow unit in near-wall turbulence," Journal of Fluid Mechanics, Vol. 225, 1991, pp. 213-240.

${ }^{9}$ Gaitonde, D. V. and Visbal, M. R., "High-order schemes for Navier-Stokes equations: Algorithm and implementation into FDL3DI," AFRL-VA-WP TR-1998-3060, 1998.

${ }^{10}$ Morgan, P. E., Visbal, M. R., and Rizzetta, D. P., "A Parallel High-Order Flow Solver for Large-Eddy and Direct Numerical Simulation," 32nd AIAA Fluids Dynamics Conference and Exhibit, No. 2002-3123, 2002.

${ }^{11}$ Lele, S. K., "Compact Finite Difference Schemes with Spectral-like Resolution," Journal of Computational Physics, Vol. 103, 1992, pp. 16-42.

${ }^{12}$ Rizzetta, D. P., Visbal, M. R., and Blaisdell, G. A., "Application of a High-Order Compact Difference Scheme to LargeEddy and Direct Numerical Simulation," 30th AIAA Fluid Dynamics Conference, No. 1999-3714, 1999.

${ }^{13}$ Visbal, M. R., Morgan, P. E., and Rizzetta, D. P., "An Implicit LES Approach Based on High-Order Compact Differencing and Filtering Schemes (Invited)," 16th AIAA Computational Fluid Dynamics Conference, No. 2003-4098, 2003.

${ }^{14}$ Gaitonde, D. V. and Visbal, M. R., "Padé-Type Higher-Order Boundary Filters for the Navier-Stokes Equations," AIAA Journal, Vol. 38, No. 11, November 2000.

${ }^{15}$ Kawai, S. and Lele, S. K., "Localized artificial diffusivity scheme for discontinuity capturing on curvilinear meshes," Journal of Computational Physics, Vol. 227, 2008, pp. 9498-9526.

${ }^{16}$ Kawai, S., Shankar, S. K., and Lele, S. K., "Assessment of localized artificial diffusivity scheme for large-eddy simulation of compressible turbulent flows," Journal of Computational Physics, Vol. 229, 2010, pp. 1739-1762.

${ }^{17}$ Morgan, B., Duraisamy, K., Nguyen, N., Kawai, S., and Lele, S. K., "Flow physics and RANS modelling of oblique shock/turbulent boundary layer interaction," Journal of Fluid Mechanics, Vol. 729, 2013, pp. 231-284.

${ }^{18}$ Lund, T. S., Wu, X., and Squires, K. D., "Generation of Turbulent Inflow Data for Spatially-Developing Boundary Layer Simulations," Journal of Computational Physics, Vol. 140, 1998, pp. 233-258.

${ }^{19}$ Urbin, G. and Knight, D., "Large-Eddy Simulation of a Supersonic Boundary Layer Using an Unstructured Grid," AIAA Journal, Vol. 39, No. 7, July 2001, pp. 1288-1295. 


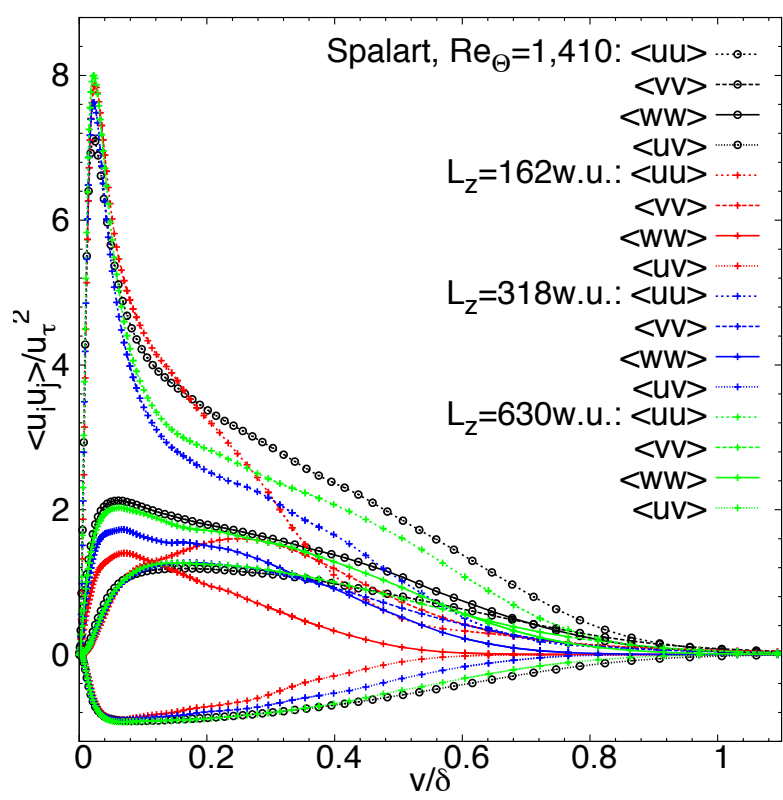

(a) Across the boundary layer

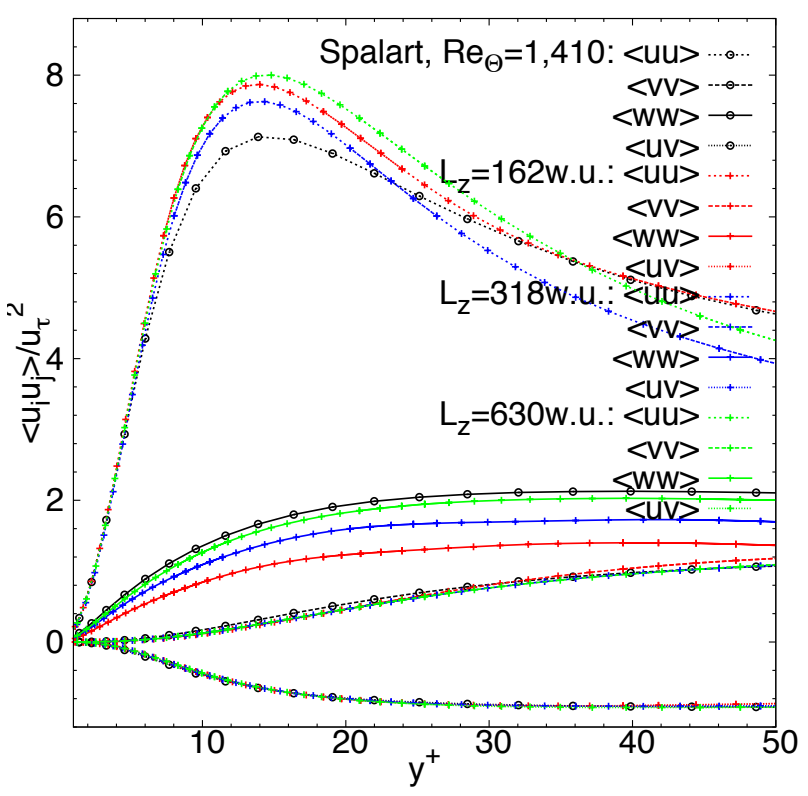

(b) In the viscous near-wall region

Figure 9. Reynolds stresses for different span widths, normalized by friction velocity, at Re $\theta=1,400$, compared with DNS results of Spalart ${ }^{1}$

${ }^{20}$ Morgan, B., Larsson, J., Kawai, S., and Lele, S. K., "Improving Low-Frequency Characteristics of Recycling/Rescaling Inflow Turbulence Generation," AIAA Journal, Vol. 49, No. 3, March 2011, pp. 582-597.

${ }^{21}$ Morgan, B. E., Large-Eddy Simulation of Shock/Turbulence Interactions in Hypersonic Vehicle Isolator Systems, Ph.D. thesis, Stanford University, 2012.

${ }^{22}$ Moin, P. and Mahesh, K., "Direct Numerical Simulation: A Tool in Turbulence Research," Annual Review of Fluid Mechanics, Vol. 30, 1998, pp. 539-578.

${ }^{23}$ Moser, R. D., Kim, J., and Mansour, N. N., "Direct numerical simulation of turbulent channel flow up to $\operatorname{Re}_{\tau}=590$," Physics of Fluids, Vol. 11, No. 4, 1999, pp. 943-945. 